\title{
Feminismo(s): hibridaciones frente a diferencialismos (Feminism(s): Hybridation versus differentialisms)
}

\author{
OÑATI SOCIO-Legal SERIES Volume 10, IsSue 1S (2020), 135S-159S: THE FOURTH WAVE OF \\ FEMINISM: FROM SOCIAL NETWORKING AND SELF-DETERMINATION TO SISTERHOOD \\ DOI LINK: HTTPS://DOI.ORG/10.35295/OSLS.IISL/0000-0000-0000-1115 \\ RECEIVED 14 JANUARY 2019, ACCEPTED 25 NOVEMBER 2019
}

\section{BARBARA BIGLIA*ID}

\section{Resumen}

En este artículo se propone una reflexión sobre algunos de los sentidos que la palabra feminismo puede adquirir para las activistas de movimientos sociales. En un momento histórico en el cual el término es usado con una cierta ligereza, analizaremos las influencias de los condicionantes sociales en la autoidentificación como feminista. Se toma, como punto de partida, la opinión de activistas italianas, chilenas y del Estado español entrevistadas en la primera década de este siglo en el marco de la tesis doctoral Narrativas de mujeres sobre las relaciones de género en los movimientos sociales mixtos. El análisis de su vivencia y percepción sirve como estímulo para un debate que apueste por un feminismo que se desplace desde una lógica diferencialista, hacia una visión que, sin negar las particularidades, reconozca y ponga en valor las hibridaciones y el trabajo en red.

\section{Palabras clave}

Feminismo(s); movimientos sociales; hibridaciones; networking

\section{Abstract}

This article proposes a reflection on some of the meanings that the word feminism may have for social movement women's activists. At a historical moment in

\footnotetext{
* Doctora en psicología (UB, 2006) con mención de doctorado europeo, es actualmente Profesora Agregada Serra Hunter en el Departamento de Pedagogía de la URV. Desde el principio de su trayectoria investigadora ha trabado bajo el prisma de la perspectiva de género/feminismo, tratando temáticas diversas como los procesos de subjetivación y la influencia en ellos de los roles de género; las movilizaciones colectivas y los movimientos sociales; los procesos de producción de conocimiento y la epistemología feminista; la docencia virtual y la pedagogía (ciber)feminista; las violencias de género, etc. Fundadora del SIMReF en 2008 es también miembro del Grupo de Investigación Consolidado Interuniversitario GREDI y del Institut Interuniversitari d'Estudis de Dones i Gènere. Ha sido Visitor Researcher en la Manchester Metropolitan University (2004) y en el Graduate Center de la Cuy University of NY (2012). Datos de contacto: Departament de Pedagogia, Universitat Rovira i Virgili, Fac. Ciències de l'Educació i Psicologia. Carretera de Valls, s/n, 43007 Tarragona. Dirección de email: barbara.biglia@urv.cat Página web: http://www.simref.net/barbara-biglia/
} 
which the term is used with a certain lightness, we will analyze the influence of social factors in women's self-identification as feminists. The point of departure is the opinion of Italian, Chilean and Spanish activists interviewed at the beginning of the century in the framework of the doctoral thesis Women's narratives on gender relations in mixed social movements. The analysis of their experience and perception serves as a starting point to bet on a feminism that moves from a differentialist logic towards a vision that, without denying the particularities, recognizes and valorizes hybridations and networking.

Key words

Feminism(s); social movements; hybridation; networking 


\section{Table of contents}

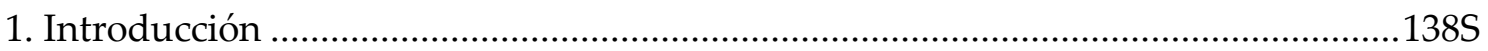

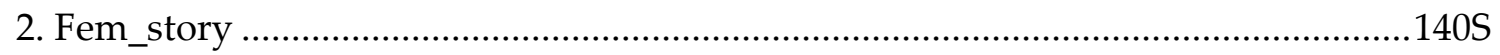

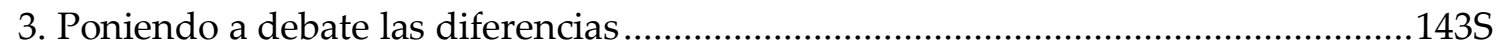

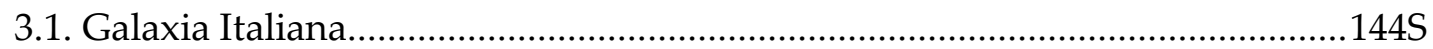

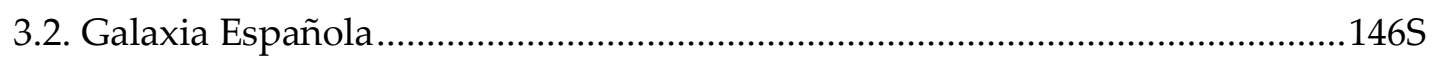

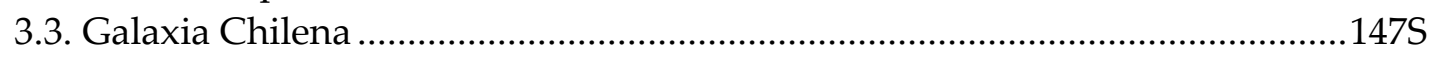

4. Alianzas, hermandades, hibridaciones: ¿políticas a-identitarias y/o múltiplemente

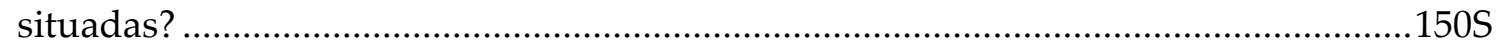

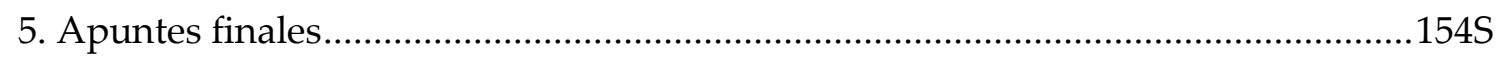

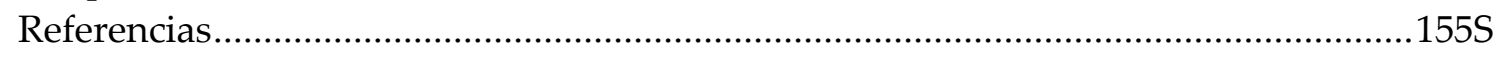




\section{Introducción}

Estamos en un momento histórico en el que la palabra feminismo parece suscitar simpatías por parte de amplios sectores, sociales, políticos y periodísticos. Las concurridas y poderosas reivindicaciones feministas de los últimos años no han dejado indiferentes a nadie, por lo que tanto los programas políticos como los medios de comunicación hacen amplia referencia a estas, exaltándolas o denigrándolas.

Por ejemplo, en 2018, el recién estrenado gobierno del PSOE (Partido Socialista Español) se autodeclara feminista, haciendo énfasis en el número de mujeres a cargo de ministerios. Unos meses después los ultraderechistas de $\mathrm{VOX}^{1}$ entran en el parlamento andaluz con un programa que señala directamente al feminismo como enemigo y propone entre otras cosas derogar la ley de violencia de género y el derecho al aborto garantizado en la ley de derechos sexuales y reproductivos. Al finalizar el año el Proyecto Cuéntalo recibe una gran atención mediática, presentando el análisis de 150.000 tuits en los que las mujeres, bajo la etiqueta de \#cuéntalo, denunciaron públicamente abusos sexuales sufridos en su vida ( $\mathrm{http}$ ://proyectocuentalo.org/).

Todos estos elementos influyen en que amplios sectores de jóvenes estén volviendo a autodefinirse feministas, como ha ocurrido a las chicas del colegio Dominiques de Barcelona que, después del 8 de marzo del 2017 han realizado un camino de empoderamiento que las ha llevado, entre muchas otras cosas, a producir un documental contando las experiencias de las violencias vividas (37Historias, https://ca.goteo.org/project/37-histories).

Sin embargo, nos podemos preguntar, ¿cuáles son los sentidos del feminismo hoy en día y cómo se configura la autoidentificación como feminista? Para reflexionar sobre esta temática tomo, como punto de partida, uno de los análisis realizados en mi tesis doctoral (Biglia 2005) sobre la influencia de los contextos histórico, sociales y políticos en la autoidentificación como feministas. El tema de esta investigación -las relaciones de género en los movimientos sociales- no surgió de una inquietud intelectual sino de la vivencia, como activista, de una problemática todavía poco reconocida. ${ }^{2}$ En ella, el entramado metodológico supuso un proceso de crecimiento importante, en el que con la experiencia de los años, es fácil detectar errores y simplezas; a medida que fui experimentando las limitaciones del método científico clásico me di cuenta de la importancia de buscar otros referentes para conseguir que todo el proceso de investigación fuese coherente con el enfoque teórico y el compromiso político con el cual me acercaba a la temática en examen. En este camino encontré las epistemologías feministas y me entusiasmé con la propuesta del conocimiento situado de Haraway (Biglia y Bonet 2017). Coherentemente con esto, y a sabiendas de que se trataba de una temática de estudio novedosa, mi interés fue aprender de las mismas protagonistas y poner en valor sus conocimientos expertos. Las temáticas específicas sobre las que concentré mi atención en el texto de la tesis se fueron por lo tanto configurando de forma

\footnotetext{
1 "Vox" no es una sigla, sino el nombre del partido que es la locución latina de "voz".

${ }^{2}$ En realidad, ya algunos años antes de empezar el doctorado, Eva Forrest, a la que estaba entrevistando para mi trabajo final de licenciatura, me preguntó por qué no estaba investigando sobre cuestiones de género si estas permeaban mi experiencia vital y activista. Esta pregunta me estimuló a tomar la decisión sobre mi tema de tesis.
} 
emergente, a partir de los aprendizajes que me proporcionaron los encuentros con las informantes.

Lo mismo ha ocurrido con las narrativas que presento en este artículo, a partir de treinta y una entrevistas a activistas de movimientos sociales mixtos a principios del siglo XXI, con una edad que varía entre los 21 y 74 años (media de edad, 38). Las protagonistas de este trabajo pertenecen a movimientos sociales variados entre los cuales, el movimiento autónomo, libertario, de toma de tierras, mapuche, okupa etc. La muestra se ha autoconfigurado a través de la técnica de la bola de nieve ya que, dada la especificidad de estos movimientos en aquellos años, la reticencia a hablar con investigadoras era bastante importante. Su participación fue posibilitada y mediada por mi propio activismo en Italia y en el Estado español que me permitió acceder a amigas de amigas activas en movimientos sociales mixtos. ${ }^{3}$ No se pretende en ningún caso presentar sus voces como muestra representativa de las activistas en estos contextos geopolíticos, sino reconocer sus conocimientos situados como valiosos para la comprensión de la realidad. La idea, en este sentido, es producir teoría desde y con los movimientos sociales en lugar de sobre ellos (Biglia 2007).

En este artículo desarrollo un acercamiento a las diferentes percepciones y vivencia de las realidades, debidas tanto al contexto socio histórico, político y cultural, como a las experiencias personales de las protagonistas. Este análisis, como explicaré en los apartados finales de este escrito, me sirvió como referente y estímulo para apostar hacia un desplazamiento del feminismo desde una lógica diferencialista, a una visión que, sin negar las particularidades, empuja hacia la posibilidad de hibridaciones y la necesidad de un trabajo en red. Es este último elemento, que surgió como resultado del análisis de las palabras de la entrevistadas, el que hace relevante volver a poner este debate en circulación en el contexto de este monográfico, aunque mejor no adelantemos contenido.

Para realizar este camino me he servido de la construcción de sentido que realizamos, tanto a nivel teórico como en la práctica, de la palabra feminismo. ¿De qué manera nuestra particular-colectiva interpretación del feminismo nos hace vivir las relaciones generizadas? ¿Cómo influyen las diferencias culturales, históricas y de movimiento en esta percepción? ¿De qué manera las etiquetas tienden a separar nuestros quehaceres? ¿Cómo romper con las falsas jerarquizaciones de un "primer mundo" igualitario frente a un "tercer mundo" en el que las mujeres son sumisas? Estas son algunas de las preguntas a las cuales quiero acercarme.

Dedico la segunda sección de este paseo a un breve excursus teórico sobre los feminismos (principalmente occidentales), consciente de que este camino será incompleto dado que se irá topando con la limitación de quienes son las que pueden producir y difundir teoría. De hecho, como bien afirma Barbara Cooper (1995), con demasiada frecuencia, desde las posiciones de poder de las intelectuales feministas euro americanas, se define el o los feminismos sobre la base de criterios relativos a nuestro entorno cultural. Esto lleva a analizar los feminismos "de otros lugares" con gafas deformantes, y a aplanar sus sentidos a los nuestros (para una óptica menos eurocéntrica, se aconsejan, entre otros, Davies 1987, Cooper 1995, Peoples' Global Action -PGA- 2001). En el tercer apartado,

\footnotetext{
${ }^{3}$ Agradezco en este sentido la confianza de todas ellas, la ayuda de las fantásticas gatekeepers a la hora de contactarlas y los largos debates con mis queridas amigas chilenas, especialmente con Francia, para ayudarme a comprender y contextualizar las experiencias y aportaciones de las mujeres entrevistadas.
} 
intento romper la barrera de lo teórico para que el feminismo se corporeice en las prácticas activistas de quienes no llegan generalmente al estatus de productoras de teorías reconocidas. Aquí navego entre los sentidos del término "feminismo" reflexionando sobre cómo los factores sociopolíticos y personales influyen en su constitución. Finalmente, se pretende poner en diálogo estas vivencias para facilitarnos la comprensión y la puesta en común de los debates. La última sección, recogiendo los hilos trazados a lo largo del artículo, quiere presentar la apuesta política de las hibridaciones y del networking, como manera de tejer colectivamente.

\section{Fem_story}

¿Quién y cómo se define feminista y qué prácticas pueden ser consideradas feministas? Y más aún: ¿Cuándo empezó el feminismo? Si por feminismo se entiende la rebelión frente a las discriminaciones de género, sus inicios son probablemente contemporáneos a las discriminaciones mismas ya que todo poder produce resistencias. $\mathrm{Si}$, en cambio, queremos buscar antecedentes en espacios de insumisión más colectiva, "puede afirmarse que ha sido en los periodos de la Ilustración y en los momentos de transición hacia formas sociales más justas y liberadoras cuando ha surgido con más fuerza la polémica feminista" (De Miguel 1995, 249). De hecho, la historia está llena de protestas capitaneadas por mujeres trabajadoras que, si bien en muchos casos no reivindicaban explícitamente el cese de las discriminaciones de género, pueden considerarse como predecesoras del feminismo, en tanto que no dudaron lo más mínimo en movilizarse de manera autónoma actuando contra los preceptos de género.

Es, sin embargo, con la Revolución Francesa, a través de explícitas peticiones de igualdad sexual, que se considera que empieza la articulación del feminismo moderno.

Finalmente, "en el siglo XIX, el siglo de los grandes movimientos sociales emancipatorios, el feminismo aparece, por primera vez, como un movimiento social de carácter internacional, con una identidad autónoma teórica y organizativa" (De Miguel 1995, 255). Esta lucha de las sufragistas por el derecho al voto y la emancipación de las mujeres configuran lo que se suele identificar como la primera ola del feminismo aunque, en mi opinión, esta atribución póstuma tiende a no reconocer las importantes movilizaciones anteriores y podría ser tildada de clasista y eurocéntrica.

Fue en los años 60 y 70 del siglo XX, en el seno de un clima de efervescencia social generalizado que emerge en muchos países de Europa y de América, que tiene inicio la que se considerará como la segunda ola del feminismo. Propone una nueva forma de pensar que quiere distanciarse de manera directa de las anteriores "con la crítica al hecho emancipatorio tal como se había fijado en la tradición de la cuestión femenina" (Fraire y Frabotta 2002, 10). Esta incluye prácticas muy distintas e innovadoras, entre las cuales hay que destacar la importancia de los grupos de sólo mujeres. Estos grupos no mixtos eran vividos de una forma extremadamente transformadora para sus participantes. Sin embargo, esta adquisición de autonomía no ha sido indolora y ha llevado a varios enfrentamientos, y muchas militantes de partidos y grupos de izquierda "se separaron de las organizaciones mixtas en conflicto con los hombres por el derecho a organizarse autónomamente" (Roseneil 2000, 143).

En general, podemos afirmar que, si bien las practicas del MF (movimiento feminista) occidental han sido bastante compartidas, estas se han basado y han originado teorías 
muy variadas que han llevado en ocasiones a debates y oposiciones ideológicas en el transcurrir de los años. Feministas de la diferencia versus feministas de la igualdad, integracionistas versus separatistas, heterosexuales versus lesbianas, TERF (Trans Exclusive Radical Feminist) versus transfeministas, abolicionistas versus proderechos etc...

En el nuevo siglo nos encontramos en una situación de transición, ya que, aunque el fin de la segunda ola del feminismo no ha sido reconocido oficialmente, y desde muchos ámbitos se evidencia cómo las transformaciones que ha atravesado el feminismo han sido de tal alcance que se ha producido un cambio de ola.

Sin embargo, hay quien consideran que el feminismo ya no tiene razón de ser, por ejemplo, las que, declarándose neofeministas, proponen la adhesión completa a valores heteropatriarcales (Bel Bravo 2004) y quienes creen que lo queer, desarticulando los géneros, representa una superación del feminismo.

En este contexto, aunque pueda ser relativamente sencillo explicar por qué y de qué manera personal una se siente feminista, surge un sinfín de problemas cuando se intenta definir qué significa ser feminista en un sentido más general. La multiplicidad de significados que ha ido adquiriendo la palabra feminismo, hace que cualquier opción resulte inevitablemente simplificadora. De hecho, para las generaciones más jóvenes, feminismo se declina preferiblemente en plural: feminismos. Sin embargo, desde un compromiso político con el feminismo, el no ser crítica con algunos de los usos del concepto es claramente imposible.

No obstante, la idea de inventar una nueva palabra es, frustrante, porque implicaría la renuncia a la larguísima historia de la micro- y macro- subversión diaria de las mujeres. Asimismo, la invención de nuevos términos no siempre nos permite salir de las contradicciones. Por ejemplo, el uso alternativo de posfeminismo se asocia con dos realidades antagónicas. Por una parte, se utiliza la acepción que propone Preciado (2003): “Las multitudes queer no son post-feministas porque no quieran o deseen actuar sin el feminismo. Al contrario. Son el resultado de una confrontación reflexiva del feminismo con las diferencias que éste borra para favorecer un sujeto político 'mujer' hegemónico y heterocentrado". Por otra, tal como explica Gamble (2001), se asocia con la negación toutcourt del feminismo a través de dos diferentes representaciones. Una enmarcada en la reinterpretación (cuando no directamente creación) por parte de los medios de comunicación de fenómenos artísticos como las Spice Girls, en los que jóvenes mujeres asumen imágenes femeninas estereotipadas (y sensuales) mezcladas con actitudes sexistas masculinizadas. Otra, en los discursos de teóricos neoliberales y/o neoconservadores que, manteniendo un fuerte sesgo heterosexista y partiendo de la negación de la victimización de las mujeres, descalifican los análisis feministas de fenómenos como las violaciones (Gamble 2001).

Al mismo tiempo, algunos de los imaginarios que el uso del concepto feminismo evoca están extremamente alejados de los que, en mi opinión, son posibles sentidos del término $\mathrm{y}$, en casos extremos, pueden incluso reforzar prácticas heterosexistas y/o patriarcales. Así que podemos preguntarnos, ¿cuáles son los feminismos? ¿Qué tienen en común y que los diferencia? 
Sin dar una definición cerrada, me atrevo a afirmar que los feminismos son ontologías del mundo, y el feminismo un proyecto articulado y polimórfico de vida colectiva y, en este sentido también, o quizás sobre todo, una política. El feminismo es, así, "un modo de sentir, de ser, de actuar y reaccionar. Es una llave de lectura del mundo sin las anteojeras. Es un nervio ardiente constantemente descubierto, que ayuda a reconocer y descubrir potentes estereotipos" (Cirant 2005).

Considerando que Feminism is for everybody (hooks 1984/2000), la crítica de las feministas negras/no blancas y/o lesbianas durante la mal llamada segunda ola del feminismo en torno a la construcción discriminatoria de mujeres como categoría unificada (para una descripción: Nicholson 1997, Meyers 1997), tiene que ser ampliada para la redefinición de políticas feministas. Esta necesidad persiste en tanto que las agendas y prioridades feministas todavía tienden a ocultar las necesidades y las agencias que están emergiendo desde arenas geopolíticas o grupos minorizados (Cooper 1995).

De hecho, aun cuando podemos reconocer la multiplicidad, fragmentariedad, nomadismo, ciborgidad de las identidades involucradas en la construcción de las subjetividades feminizadas, incluso en las políticas feministas se tiende a reproducir un discurso que quisiera ser universal representando, en realidad, sólo una particular visión de la realidad. "Por ejemplo, la petición de mayor protección policial que suscribirían muchas mujeres y algunos grupos feministas, puede suponer para trabajadoras sexuales y mujeres migrantes -sobre todo sin papeles- más que garantía de seguridad, una amenaza de acoso y, en ocasiones, de agresión y expulsión. Evidencian, así, diferencias cruciales sobre qué constituye una prioridad política dentro del feminismo y qué estrategias seguir para alcanzarlas" (Escalera Karakola 2004). Esto, en mi opinión, nos lleva a lo que se configura hoy en día como uno de los principales desafíos de las políticas feministas: el encontrar maneras de luchar por nuestras legítimas demandas situadas sin oscurecer otras necesidades. "Debemos ver la política feminista (y otras formas de democracia) como formas de políticas de coalición en que las diferencias entre las mujeres son reconocidas y se les da voz, sin fijar los términos de la coalición a partir del 'quienes' somos sino a partir de lo que queremos obtener" (Yuval-Davis 1993, 4).

La importancia de la recuperación de los procesos colectivos es puesta de manifiesto también por Erica Burman (2003) que evidencia cómo el extremo valor que desde el feminismo se ha dado a las prácticas introspectivas ha producido (como efecto secundario) la reducción del poder de las colectividades. O sea, de alguna manera, nos hemos centrado demasiado en resaltar nuestras peculiaridades y especificidades y no nos hemos dedicado lo suficiente a la construcción de alianzas. Así, sigue Burman, si el feminismo posmoderno ${ }^{4}$ ha ofrecido herramientas extremamente potentes en contra de la cooptación y despolitización del lema feminista lo personal es político, por otra parte, ha producido un desplazamiento hacia el self que ha tenido como consecuencia un renovado interés por la terapia que ha favorecido la construcción de nuevas patologizaciones. De todas maneras, como analizaremos más en profundidad después, hay que contestar a la llamada a la acción colectiva sin idealizar la comunidad, como se

\footnotetext{
4 Siguiendo a Piedra Guillén (2003,43-4), el feminismo posmoderno es aquel que "plantea la necesidad de crear rupturas centrales y determinantes con el pensamiento occidental, masculino, moderno, falocéntrico y dominante, que ha estado presente en el desarrollo de la humanidad, en especial en la sociedad occidental".
} 
hizo desde algunas prácticas feministas, para prevenir el riesgo de homogeneizar o descuidar las diferencias (Young 1990).

\section{Poniendo a debate las diferencias}

¿Cómo influyen las diferencias socio históricas en la vivencia del feminismo? Cuando hablamos de feminismo, ¿entendemos todas lo mismo? ¿En qué puntos nuestras experiencias se pueden cruzar?

Para acercarme a esta complejidad he decidido analizar las respuestas a la pregunta de si se sentían o no feministas y qué sentido daban a este término las treinta y una mujeres (siete españolas, 11 chilenas y 13 italianas) que he entrevistado en mi tesis.

Es interesante reflexionar comparativamente sobre los contextos geopolíticos de las entrevistadas que comparten algunas características relevantes. En primer lugar, la cultura latina, caracterizada por elementos como la importancia de la familia nuclear y el peso de la religión católica en los múltiples aspectos de la vida. Seguidamente, la fuerte oposición a los primeros feminismos por parte de los partidos y grupos de izquierda de los respectivos países. Como tercer elemento similar, mencionar las experiencias de profundos cambios sociales (aunque en épocas diferentes): el gobierno de Allende en Chile; la importancia de las y los partisanos y el comunismo en el momento de la derrota de los alemanes en Italia; la Guerra Civil y las experiencias de colectivización en el Estado español. En todos estos momentos, así como en muchos otros, la participación política y activista de las mujeres fue muy alta, aunque hubo mucha resistencia a reconocerlo. Por ejemplo, "las mujeres [en Italia] mantuvieron una resistencia armada como los hombres, [pero] en las manifestaciones por la liberación encontramos la ausencia de muchas partisanas, porque sus compañeros varones temían que su presencia pudiera ser leída en otros términos y consideradas, como dice una testigo, 'las putas del grupo'"' (Koch, en Il colpo della strega 1995, 35).

Finalmente, los tres lugares están marcados por largos periodos de dictadura caracterizados por una fuerte retórica nacionalista en la que el rol de las mujeres como retransmisoras de los valores de la comunidad madres cariñosas y cuidadoras, símbolo encarnado del territorio nacional- ha sido exaltado. En estas etapas se produjo un claro retroceso de las libertades de elección individuales y colectivas de las mujeres y una separación entre "buenas" y "malas". Esto porque si "las mujeres, lo femenino y las figuras de género, han afianzado tradicionalmente el imaginario nacionalista, ciertas mujeres, prostitutas y lesbianas se están viendo disciplinadas y expulsadas de la escritura del guión de la nación" (Alexander y Mohanty 2004, 155-6).

Estas comunalidades podrían hacernos suponer una autoidentificación parecida respecto al feminismo por parte de las activistas entrevistadas. En cambio, como podemos apreciar en el gráfico 1, hay unas tendencias diferentes. Así, sólo una "chilena" se ha declarado feminista, y las "españolas" parecen las menos reticentes a autodefinirse como tales. Estas informaciones adquieren significado en el momento en el que nos detenemos en ver qué es lo que realmente afirman respecto al sentido del concepto feminismo. Vamos a explorarlo intercalando reflexiones históricas sobre los tres contextos con las palabras de las entrevistadas. 


\section{GRÁFICO 1}

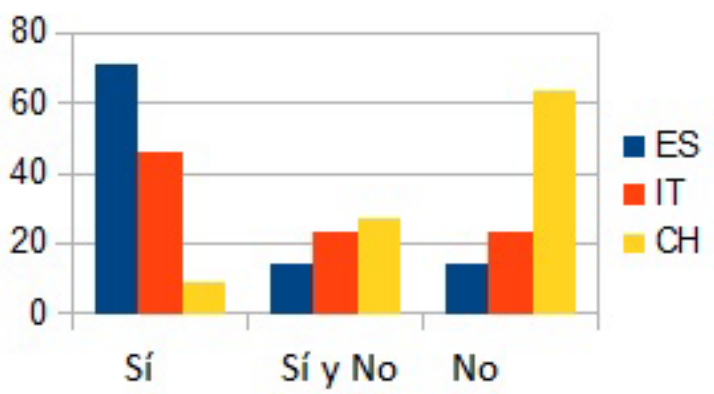

Gráfico 1. ¿Te consideras feminista?

(Fuente: Elaboración propia.)

\subsection{Galaxia Italiana}

En Italia, la dictadura ${ }^{5}$ de Mussolini fue relativamente breve (desde el 1922 al 1945) y acabó gracias a un fortísimo movimiento partigiano y la intervención del Ejército norteamericano. ${ }^{6}$ En esta época, "las mujeres eran protagonistas, en el centro y en el sur de Italia, de las luchas por los derechos de las jornaleras, con la ocupación de las tierras. Ya en 1944 se habían realizado enfrentamientos entre las fuerzas del orden y mujeres que protestaban por la reforma agrícola para la eliminación de aquella costumbre feudal según la cual las aparceras tenían que prestar servicio doméstico y de cocina gratuito a los patrones, así como regalar pollos, huevos y conejos" (Mariani, en Il colpo della strega 1995, 24). Estas expresiones de protesta colectiva no fueron disminuyendo, y después de la posguerra y de la reconstrucción de la nación, en una época de boom económico, las mujeres fueron parte integrante y activa de fuertes movimientos sociales. La fuerza de los MS (movimientos sociales) en la Italia de los 70 ha sido de gran alcance. Sin embargo, en su seno, la relación con las crecientes reivindicaciones de las mujeres fue extremamente conflictiva. Unas cuantas decidieron bastante pronto formar un MF completamente autónomo de los movimientos mixtos mientras que otras creyeron oportuno seguir llevando las luchas feministas dentro de los grupos mixtos. En muchos casos, sin embargo, la lucha desde dentro llevó a conflictos muy intensos que produjeron rupturas, como ocurrió también en la cercana Alemania (Ferree y Roth 1998). Simultáneamente, dentro del MF se fueron conformando, al menos, dos líneas.

Las que decidieron apostar por la acción directa de manera independiente de los varones:

En aquellos años practicábamos varias formas de lucha: desde denuncias a la prensa y a la magistratura, a los diferentes parlamentarios, hasta la ocupación de las comisarías, de las sedes de los periódicos, a la interrupción de actos públicos, sit-in delante del palacio de justicia o de las diferentes cárceles. Para mí esta ha sido una militancia feminista porque la mayoría de la asociación eran mujeres (Silvia) ${ }^{7}$

\footnotetext{
${ }^{5}$ Benito Mussolini, después de llegar al poder por la fuerza en 1922, se hizo elegir para dar una apariencia de democracia.

${ }^{6}$ Intervención que dejó secuelas importantes en el territorio, véase por ejemplo Ganser 2005.

${ }^{7}$ Las citas con nombres de pila, ficticios para garantizar el anonimato, corresponden a las entrevistas de la tesis.
} 
Las que se encerraron en unos círculos teóricos siempre más autorreferenciales: "la sofisticada reflexión teórica que lleva a cabo el pensamiento de la diferencia se aleja progresivamente del movimiento político de las mujeres. La propia necesidad de elaborar teóricamente la política de lo simbólico y delimitar sus diferencias respecto a otros modos de entender la política, convierte progresivamente el pensamiento de la diferencia en un pensamiento académico, cerrado en sí mismo" (Gómez 2004, 117).

Las primeras sufrieron la fuerte represión que a finales de los 70 y principios de los 80 llevó a la casi total desaparición de generaciones de activistas, acompañada por la cooptación de algunas de ellas dentro de los entramados institucionales.

Esperábamos cambiar el mundo y, desafortunadamente, no ha cambiado. (Simona)

Las segundas se fueron progresivamente encerrando en su pirámide dorada llevando otras a rechazar el feminismo como algo exterior y alejado, inútil para sus luchas:

... nosotras venimos, o por lo menos yo, de una familia proletaria, o sea, si feministas son las radicales chic, no; si feminista quiere decir que yo he combatido, que realmente he querido lo que son ahora, entonces sí. (Teresa)

Si el feminismo es una etiqueta, no yo no creo [que sea feminista], yo quiero ser considerada sólo como una mujer y creo que si pudiera cambiar querría ser de nuevo una mujer, pero el feminismo como etiqueta no me sirve. (Francesca)

No, no me considero feminista (...), o sea, se tendría que entender qué es el feminismo, porque es un término bastante amplio. No, no me considero feminista, sin embargo, estoy orgullosa de ser una mujer. (Maria)

Me siento partícipe de la que ha sido la lucha de las mujeres de los años 70, pero me siento lejana de las feministas que buscan la paridad como única finalidad y sin estar dentro de una lucha global. (Antonella)

Ambas tendencias llevaron en los años 80 a una situación caracterizada por escasas relaciones intergeneracionales, tanto que, como resalta Stefania, maestra de escuela:

... las chicas no ven [el sexismo en la publicidad] como algo que concierne a su sexo, por lo tanto (...) están un poco sumisas ante ello. Nosotras nos enfadábamos y ellas no. Hay esta separación con las más jóvenes.

Así las jóvenes que se acercaban al feminismo desde el movimiento en los años 80 y principios de los 90 se encontraban sin referentes:

...que palabra tan grande, no, no sé, no creo, o sea, quién sabe. Respecto a teoría del feminismo y de movimiento feminista conozco muy poco; si tuviera que contestar delante de un compañero diría que sí, y me dirían que es verdad, o sea me considero una mujer con conciencia (...). (Roberta)

y con una fuerte hostilidad de parte del movimiento mixto a causa de las escisiones de las décadas precedentes: "Con frecuencia tengo la sensación de que definirme feminista, en lugar de favorecer la relación con otras mujeres, la hace más difícil” (Sara, en Magaraggia et al. 2005, 33).

Hostilidad presente en diferentes espacios vitales: "Entre las experiencias que las mujeres viven y las maneras en las que están acostumbradas a pensarlas y contarlas, hay un espacio opaco e inhabitado: es el espacio de lo político; todo, inclusive la denuncia, ocurre de manera privada e individual. Los medios están repletos de discursos sobre lo 
'privado': mientras se pueda hacer y dar espectáculo, que se hable de ello. Pero cuando entra en juego la propia vida real, lo privado se vuelve un tabú. Al primer estertor del conflicto, se alza la barricada: por favor, ¡no hagas una cuestión política!" (Cirant 2005).

Para salir de este impasse sin renunciar a lo político, algunas intentan inventarse nuevas terminologías que, rindiendo homenaje al feminismo, faciliten la conexión con la realidad de finales de los 90, pero no es tarea fácil:

Hace años me decía 'femminiana' dado que no era una feminista de los años setenta, de este movimiento transversal, era una evolución, corporeizaba en mi realidad mi manera de ser feminista. (Federica)

Y parece que unos cuantos años después sigue siendo complicado, como muestran las palabras de las compañeras de Assalti: "Nosotras, en lugar de definirnos feministas, nos llamamos 'femminielle' en el sentido de post-todo y particularmente de post-ismos, post-istas" (Assalti, en Magaraggia et al. 2005, 32).

Estas tendencias son, a mi entender, expresión de un nuevo giro de los colectivos y grupos feministas surgidos alrededor del nuevo milenio. Afortunadamente, tal y como muestran los testimonios presentados por Magaraggia, Martucci y Pozzi (2005), parece que haya una mayor capacidad-posibilidad de mantener una relación menos conflictiva con el pasado, entrando en diálogo con él, y recuperando una indispensable visión histórica. Probablemente esto se puede asociar también con un renovado interés por el debate por parte de las "feministas históricas".

\subsection{Galaxia Española}

En el Estado español la dictadura de Franco fue la más larga de las tres, durando casi 40 años (1936-1975) y terminando mucho más tarde que la de Mussolini. "Tras la guerra civil, el régimen de Franco retorna al código civil napoleónico de 1889, con la abolición del matrimonio civil y del divorcio y la prohibición del uso de métodos anticonceptivos y del aborto. Derechos todos ellos que no se recuperan hasta la década de los 80" (Bosch et al. 2001). Por esto, la ola de protesta social que recorrió Europa a partir de finales de los 60, no generó en el Estado español movimientos de masas donde cualquier movilización recibía una represión muy directa y las diferentes reivindicaciones cristalizaban en torno a la lucha antifranquista. De hecho, muchas activistas y militantes tuvieron que vivir su activismo en la clandestinidad o migrar hacia otros lugares. "Será a partir de la muerte del General Franco y los cambios políticos que conocemos como transición democrática cuando el movimiento feminista español se irá organizando" (Bosch et al. 2001). Después de la transición, el Estado español se encontró contacto directo con unas realidades deliberación sexual y femenina que no habían seguido un proceso lineal interno, porque, aunque muchas feministas habían trabajado para ello, no hubo una lenta y progresiva dinámica de cambio social colectivo, así que muchas personas percibieron un salto hacia otra realidad. Probablemente por esto, muchas chicas tienen dificultad para definirse feministas, o al hacerlo consideran importante especificar que esto no significa estar en contra de los hombres:

Marta: -Vaya, que estoy rodeada de feministas pero que luego a la hora de... ¿feminista? ¿Eres feminista? No sé, creo que tengo un poco de incultura del tema por desconocimiento que luego te puedo poner ejemplos trasladados a la cotidianidad y que, vale, sí y no, pero... 
B: - ¿No te consideras feminista?

Marta: -No, no. No siento, o sea defender el derecho de la mujer, esto sí: siempre, todos los días, y a todas las horas, pero no le pongo el nombre allí.

Feminismo sin excluir, que a veces se tiende a excluir a los hombres para hacer determinadas cosas. Lo que pasa es que sí es verdad que en el movimiento feminista las mujeres siempre han ido a la vanguardia, siempre han sido las que han montado los grupos y han intentado cambiar las cosas, algunas veces se han intentado algunos grupos mixtos pero casi siempre al final se han quedado las mujeres. (Angélica)

Si es creer en la igualdad entre el hombre y la mujer. pues sí, soy feminista. No soy feminista de pensar que somos superiores ni siquiera, porque hay gente que piensa que somos superiores, pues no tampoco hay que pensar en esto, pues seremos superiores en algunas cosas como ellos a lo mejor lo pueden ser en otras -por ejemplo en fuerza, yo que sé- es decir, que tampoco me empeño en decir 'pues nosotras somos más inteligentes' pues no sé, igual hasta me lo creo y todo, pero tampoco soy de las que dicen 'somos superiores', hay que demostrarles que somos superiores. (Mónica)

Finalmente, la apertura internacional a las dinámicas de género, juntamente con la presión de las activistas, llevó al gobierno a organizar espacios institucionales como el Instituto de la Mujer y sus correspondientes autonómicos. Sin embargo, "el éxito de la institucionalización del género en la femocracia o la banalización izquierdista y derechista de la radicalidad del feminismo en una suerte de código de conducta, convierten el sueño de un espacio de intervención social feminista en algo sumamente precario" (Serrano y López 2003). No obstante, muchas siguen creyendo que:

aunque la palabra 'feminista' muchas veces ha sido como muy boicoteada o desvirtuada tanto por la sociedad como masa como por los propios colectivos de izquierdas (...), es una palabra que (...) me parece muy llena de contenido y de lucha. (Verónica)

Durante la transición, la mayoría de los MS antifranquistas pierden su fuerza y no será hasta los años 90 que la protesta "juvenil" da inicio a una nueva etapa de los MS. Dentro de estos movimientos, surgieron varios colectivos feministas autónomos que sin embargo mantienen una relación frecuentemente conflictiva con las que años antes habían decidido organizarse de una manera más estructurada.

Soy feminista pero no me considero ni de ningún partido ni (...), feminista porque lucho porque la mujer esté mejor, o sea que tenga toda la libertad que quiera tener. (Sonia)

Últimamente me considero más parte del movimiento de mujeres que del MF pues creo que este último está estancado en peleas y disquisiciones teóricas y ha perdido su capacidad de impulso. (Alexia)

Finalmente, en el Estado español también parece haber un cambio de rumbo y una nueva apertura a la colaboración entre feministas con diferentes enfoques

Es decir, [hace unos años] no hacíamos nunca ninguna actividad en ninguna institución, ahora no, ahora hemos hecho cosas en la universidad, en la asociación de barrio donde quien paga el alquiler es el ayuntamiento, y prefiero; tanto en los movimientos alternativos como en las instituciones. (Micaela)

\subsection{Galaxia Chilena}

Por lo que concierne la dictadura de Pinochet en Chile, fue la más tardía (1973-1990), representando un fuerte retroceso en los procesos culturales y siendo caracterizada por 
la eliminación física y masiva de la oposición política. Hay que evidenciar que, de acuerdo con Neuhouser (1995), en los movimientos de Latinoamérica de la segunda mitad del siglo XX, en contraste con lo que se suele afirmar, las mujeres participan en mayor medida que los hombres en las movilizaciones sociales. Paradójicamente, "esto fue parcialmente facilitado por los mismos gobiernos militares, que frecuentemente no veían las actividades de las mujeres lo suficientemente peligrosas como para necesitar ser reprimidas" (Waylen 1994, 338). Así, las desiguales oportunidades de trabajo, que sitúan a las mujeres haciendo tareas en el barrio o en la comunidad (limpiando ropa, planchando...) y a los hombres saliendo en busca de trabajos externos, tienden a reforzar el rol de las madres como cabeza de familia (Neuhouser 1995). Sin embargo, estas activistas no tienden a identificarse como feministas. Es más, la imagen de las feministas que tienen es la que se produce desde una óptica heteropatriarcal: enemiga de todos los hombres que obstaculizan luchas de más elevado alcance.

Por lo menos en Chile, el feminismo, en cierto momento, se pasó como al otro lado, como un odio a los hombres y como que, no sé, igual yo creo que, yo creo que somos iguales, no, ni tan iguales, yo creo que las mujeres son mejor que los hombres en cosas, por lo mismo igual a los hombres les ha costado darle el espacio porque les gana, muchas veces les gana en muchas cosas entonces yo creo que para los hombres muchas veces es difícil darle este espacio. Pero no sé, no sé si me considero feminista de verdad, igual siempre he andado lidiando con los derechos de la mujer, retando a los locos cuando son ellos machistas y todo esto pero no sé si feminista. (Gracia)

Pero si es por la lucha como mujeres, como para que la respeten como para que salir a caminar a la calle y sentirme segura, sí, de todas maneras, pero del otro lado, como de resentirme con los hombres porque... no, como no me gusta el resentimiento que a veces he notado en feministas. (Paloma)

La palabra 'feminista' abarca, no sé, pues, un concepto muy amplio, y tú no tienes que olvidar que soy (...) dirigente mapuche, con una lucha muy grande, entonces no vamos a por una lucha simplemente para las mujeres, vamos a revindicar lo nuestro, todo lo nuestro para un solo pueblo independiente, que en este pueblo hay niños, ancianos, hombres, mujeres... (Marina)

Así, incluso cuando hacen declaraciones y llevan a cabo prácticas que serían fácilmente inscribibles dentro de las narrativas feministas, siguen rechazando esta etiqueta:

Yo creo que la igualdad de las personas, hombre y mujer es lo mismo. Mi forma de ser ha sido siempre igual, yo a un hombre lo miro igual como miro a una mujer. Sobre todo en el aspecto trabajo. De igual a igual. Y el rol político y social para mí es lo mismo. Y si yo tengo que discutir con un hombre, es tal cual. Digo lo que yo pienso. (Gr2Ch)

En eso tiene harto que ver en la formación de la casa misma, de mi familia. Mi papá, a mis hermanos los obligaba a aprender a coser, a tejer, a hacer comida. Y mis hermanos todos saben coser. Mi papá nos enseñaba a nosotras a construir, a hacer pan. Pero si yo creo que en eso nos estaba preparando para el futuro, pero nos obligaba a lustrarle los zapatos. Era una cosa extraña, porque a nosotras nos obligaba a aprender cosas de hombres y ellos de mujer, pero nosotras teníamos que atender a los hombres igual. Yo no me considero feminista para nada. Yo tengo esa concepción de que el hombre y la mujer tienen los mismos derechos, ella trabaja y el hombre trabaja. Cualquiera, el primero que llega tiene que hacer lo que está pendiente de la casa: lavar, cocinar. (Gr2Ch) 
Como queda patente en la brutalidad de la represión a la que asistimos en 2019, el proceso de transición chileno se hizo sobre la base y el modelo del español con el mantenimiento de muchos militares y políticos de la época pinochetista en los lugares de mando del estado democrático. Asimismo, "los movimientos populares jugaron un rol importante en la transición a la democracia. [Hay que analizar] en primer lugar el rol de las mujeres en el proceso de transición y, secundariamente, el impacto que tuvo la democratización en las relaciones generizadas" (Waylen 1994, 328). De hecho, los MS chilenos están caracterizados de una forma bastante diferente de los "europeos": hay fortísimas movilizaciones de los sectores populares en el contexto de lucha por los derechos básicos como la salud y un espacio para poder vivir en las que la presencia de las mujeres y el trabajo de red que ellas realizan es muy potente, y sin embargo difícilmente las temáticas de género son explícitamente trabajadas. Las activistas tienden a construir la propia militancia como una extensión de los propios roles familiares (Noonan 1995). Así lo expresa espontáneamente Laura (68 años) en su entrevista:

Laura: -Siempre fueron más mujeres que hombres, siempre, en todas partes, siempre.

B: - ¿Una lucha mucho más femenina?

Laura: -Sí claro, no pero nosotras siempre consideramos o sea a la familia, amigos, por ejemplo: no me gustan las feministas, a mí me gusta la familia o sea que estén las mujeres, el hombre y los niños...

B: - ¿Por qué no te gustan las feministas?:

Laura: -No; porque se supone que en la sociedad somos tanto el hombre como la mujer, tenemos que cumplir nuestros roles, entonces, aunque solo... no sirve a mucho. Yo creo que llegamos aquí a la tierra a cumplir, a casarnos, a tener hijos, yo creo...

B: -¿Por qué?

Laura:-O sea, yo creo en Dios, soy de izquierda pero siempre creo en Dios, pero no soy beata ni voy a misa ni estas cosas que a una le inculcan de chica... entonces, es la vida misma, que hay que crecer y multiplicarse, tener hijos, no, si no importa que una se case, la cosa es tener hijos también, no es necesario casarse.

B: -Pero hay mujeres que no pueden tener hijos.

Laura: -Claro, o sea, allí ya es otra cosa.

y así le hace eco otra activista:

Yo no [soy feminista] porque si yo tuviera, a lo mejor seguiría organizada, pero quiero formar una familia, una pareja, a lo mejor seguiría tal y cual porque me encanta la casa, los niños y no caigo dentro de los parámetros de las feministas, no, al revés, a mí me encantan los hombres, me encanta estar con ellos. (Gr1Ch)

La problemática relación de muchas activistas de MS mixtos con las feministas se ve atravesada por diferencias de clase y de educación, que lleva a códigos comunicativos diferentes así como a prácticas discriminadoras:

O sea, a ver, yo no me encuentro feminista porque yo conozco a muchas feministas y debe ser un poco por mi decepción, este grupo de mujeres, por ejemplo [de las que te contaba antes], todas feministas, muy feministas. A lo mejor es un poco por el estrago que yo siento que es un discurso vano, ahora siento yo que no soy feminista, ahora si me dicen luchar por tus derechos, reivindicarte, puede ser que sí lo sea, pero así como literalmente, no. (Gr1Ch) 
Así, las feministas no son vistas como las que participan de un activismo en la

calle, sino las que dirigen los partidos políticos:

Laura: -No, no. Sabes por qué digo esto, porque yo conozco a las feministas, entonces están metidas muchas en el partido comunista.

B.: -No todas, ¿no?

Laura.: -Sí, o sea, las que conozco yo, la que conocí, no me gustan porque están en un partido.

Es interesante notar, sin embargo, que en Chile existen grupos autónomos feministas que entienden "al movimiento feminista como el espacio que ejercita en todo acto la unión entre lo íntimo, lo privado y lo público. Sin estos tres niveles integrados terminamos siempre incompletas. Es su articulación lo que nos permite crear desarrollo filosófico con capacidad de propuesta de otra cultura" (Declaración del Feminismo Autónomo, en Las Clorindas 2002). Sin embargo, el imaginario sobre las feministas es tan persistente que incluso algunas que trabajan con ellas y se encuentran muy bien no pueden prescindir de él.

B.: -En [tu movimiento] hay feministas autónomas, ¿compartes lo que piensan?

Andrea:-Como discurso político, sí, con ellas me siento super cercana y en general con los movimientos feministas en Chile, pero tengo entendido, como generalizamos, como que a veces tengo esta sensación como que hay feministas que compiten con los hombres.

No obstante, impresionada por la potencia emocional y política de muchas de las mujeres con las que había conversado, me atreví, una vez apagada la grabadora, a comentar a Laura y a las chicas del grupo 1 que yo sí me consideraba feminista; que, como ya sabían, formaba parte de aquel sector de "intelectuales" que las habían tratado mal y, finalmente, que, en mi opinión, ellas eran entre las mujeres más feministas de las que había tenido el placer de aprender mucho. Las chicas del grupo 1 salieron con una sonora risa colectiva y me dijeron que bueno, yo era diferente, y me propusieron quedarme a comer y charlar más con ellas, que querían conocer más cómo funcionaban las cosas en Europa (cosa que hice con extremo gusto). Laura se puso roja y, con una gran sonrisa en la cara, me preguntó: “¿De verdad crees?”; en los días siguientes, ya amigas, se ofreció para hacerme de guía en las visitas a las ocupaciones de tierra.

\section{Alianzas, hermandades, hibridaciones: ¿políticas a-identitarias y/o múltiplemente situadas?}

Rather than bond on the basis of shared victimization or in response to a false sense of common enemy we can bond on the basis of our political commitment to a feminist movement that aim to end sexist oppression. (hooks 1984/2000, 46)

"Actuar colectivamente requiere algún tipo de identidad o conciencia colectiva" (Klandermans y de Weerd 2000,69) ¿Estamos realmente seguras de la limitación que implica esta afirmación? ¿Es todavía necesario, si alguna vez lo fue, explicar la movilización sobre la base de criterios identitarios? Y, más importante aún: para que se dé una movilización, ¿tenemos que agregarnos en torno a identidades? ¿Las activistas se sienten, de algún modo, unidas por una manera de ser común? 
Balasch y Montenegro (2003), apostando por unas movilizaciones post-identitarias, se muestran optimistas y creen que ya en los eventos contra la cumbre del Banco Mundial de Barcelona en 2001 se había superado ampliamente este paradigma. Sin embargo, los análisis que colocan las movilizaciones que han dado lugar al llamado "movimiento antiglobalización" en unos quehaceres repentinos y espontáneos no tienen en cuenta que muchos grupos y movimientos en estado de latencia han facilitado el subsuelo en el que estos acontecimientos han tenido lugar, han puesto a disposición energías e infraestructuras y han participado activamente en los procesos que han facilitado sus desenlaces (Biglia 2007). Y que muchos de estos grupos y movimientos tienen un trasfondo identitario, aunque probablemente muy diferente y más complejo de lo que se suele considerar (es decir, no directamente de género, de clase, etc.).

Vera Taylor (2000) evidencia la importancia de la disolución identitaria de los MS en un futuro: "Los movimientos sociales del futuro son simultáneamente resultado y causa de la disolución de las identidades compartidas locales, de parentesco, de raza, de etnia, de clase, de género. Son espejo de la fragmentación de la compleja sociedad moderna tanto por lo que concierne a sus formas deliberadamente descentralizadas, como en relación a la elección de sus plataformas y su ensimismamiento" (Taylor 2000, 227).

Futuro que, en los análisis de Reicher (2004), hay que introducir como elemento clave para poder darse cuenta de la flexibilidad de las acciones sociales, no sólo entre los diferentes contextos, sino también dentro de los mismos, y de que no puede disociarse de la agencia de las participantes; agencia que, como sugieren Balasch, Callén y Montenegro (2005) puede ser situada, en el análisis de los MS, sobre el sujeto de la acción (políticas identitarias) o sobre la acción misma (políticas no identitarias).

No podemos olvidar, sin embargo, que las identidades colectivas asumidas en los espacios de los MS no son generalmente reproductoras de las normalizadas, sino que son el resultado de una apuesta política y relacional. Además, "estas categorías [identitarias] se producen, en parte, de la interacción entre movimientos. Difuminar las unidades categoriales imputadas a los movimientos sociales sugiere que los dilemas identitarios corren alrededor de las fronteras de los movimientos así como dentro de ellos." (Ferree y Roth 1998, 628). Esto no significa, en modo alguno, negar que estas identidades puedan constituirse en una normalización dentro de los espacios minorizados, sino sólo que no son asimilables a categorías interpretativas externas a los movimientos mismos.

Finalmente, no tenemos que caer en la falacia de confundir los proyectos políticos identitarios con "las identidades colectivas [que] son irreductibles a la suma de las experiencias de los individuos. La identidad colectiva es el proceso de significación por el cual lo común de la experiencia en torno a un eje específico de diferenciación, digamos la clase, casta o religión se inviste de un significado particular" (Brah 2004, 132). Aunque en el pasado muchos movimientos hayan tendido a pedir a sus miembros la adhesión a la identidad colectiva que podían hacer referencia a un simbolismo naturalizado, esto está cambiando. La identidad colectiva puede ser mucho más fluida, estratégica y no esencialista. Así, siguiendo a Butler (1990), podemos considerar la importancia de la diferencia como condición de posibilidad y simultáneamente límite constitutivo de la identidad que hace posible su articulación e imposibilita su estancamiento. 
En palabras de las activistas-investigadoras Borio, Pozzi y Roggero (2004), "la identidad es necesaria, pero no puede sobrevivir invariable a los procesos que la han alimentado, so pena de convertirse en un freno y en un lastre. Hay que construir una identidadproceso, capaz de situarse dentro de las dinámicas de conflicto y contra la propia hipóstasis" (Borio et al. 2004, 68).

Lo que me deja perpleja en este análisis es la centralidad que se sigue atribuyendo a los movimientos como estructuras $u$ organizaciones indispensables para los procesos de transformación social, pero también como grupos que de alguna manera forjan las diferentes subjetividades que transitan por él. A mi entender, el debate que se está desarrollando desde el feminismo intenta superar esta limitación y, por esto, se configura como particularmente interesante.

Como se ha mencionado en la primera sección de este artículo, el feminismo como proceso colectivo surgió como elemento emancipatorio, llevó a la petición de un reconocimiento identitario de las "mujeres" y se fragmentó en las políticas diferencialistas (Brah 2004). En este proceso se perdió de vista que "existen múltiples formas de opresión y cualquier 'identidad común' es una ficción política (...). La construcción de coaliciones se desarrolla a partir de puntos de contacto entre diferentes individuos, identidades y movimientos (...) [. E]n las coaliciones las diversidades perduran y la solidaridad es temporal, específica y estratégica: cooperación actual para obtener ventajas comunes" (Ferree y Roth 1998, 629).

Por lo tanto, sería importante, en lugar de mantener en el centro del debate las identidades o su deconstrucción, mirar hacia la construcción de tecnologías para la trasformación social. Silvia García y Carmen Romero (2002) proponen, en este sentido, pensar en la articulación como un artefacto teórico-político, como propuesta ontopolítica.

Podemos seguir a Ngai-Ling Sum (2000) en su conciso, interesante y denso repaso de algunas de las más destacadas propuestas feministas para la superación de las fragmentaciones que conllevan las posiciones diferencialistas. La autora parte del esencialismo estratégico de Spivak, que sugiere que la identidad femenina es producida y regulada en relación con un determinado contexto y marcada por específicas coordenadas de poderes. De acuerdo con ella, "el sujeto mujer puede ser visto como un migrante conceptual cuya identidad es constituida desde condiciones externas que se mueve estratégicamente desde una forma de esencialismo a otra" (Sum 2000, 137).

Pasa por la política de los compromisos de Haraway, según la cual los sujetos no necesitan una 'esencia' para actuar colectivamente, y las diferencias vienen interpretadas como un problema creado por la lógica de la objetividad. Se desplaza, de la mano de Monthany y Andalzúa, hacia la idea de que las posiciones minorizadas y las diferencias que las habitan pueden desencadenar una guerra de posiciones desde las fronteras. Fronteras que, de acuerdo con Ong y Yuval-Davis, se trasforman desde sitios de resistencia a espacios permeables y transversales en los que las diferencias deben de ser reconocidas y dialogizadas, posición que, según Sum, puede ser practicable sólo por la élite de la comunidad epistemológica. Finalmente, reflexiona, con Sum, sobre recombinación de las políticas feministas con las gobernanzas híbridas para pasar desde las políticas de la identidad a las de la complejidad, que se refieren a "los problemas de cómo introducir orden en el caos y/o derivar orden desde el caos" (Sum 2000, 139). Es decir, subraya la 
importancia de las políticas feministas de la comprensión de tres niveles interrelacionados: el intrapersonal, las negociaciones interorganizacionales y las relaciones intersistémicas. Esto, a su modo de ver, se puede concretar en preguntarnos (entre otras cosas): “¿Qué tipo de relación yo-otro se construye en los diálogos interpersonales entre múltiples sujetos femeninos y cómo son hibridadas? ¿Implican la construcción de nuevas redes conformadas libremente para permitir a sujetos y grupos femeninos comunicarse?" (Sum 2000, 141).

La apuesta hacia la cual me decanto, gracias también a lo aprendido con las entrevistadas, es la de considerarnos subjetividades híbridas y favorecer la complejización y colaboración de las hibridaciones a través del network(ing). Network(ing) como espacio, necesidad, proceso, resultado, limitación, punto de partida, opción política y mucho, mucho más. Network(ing) como un cyborg, que no puede prescindir de las tecnologías para existir y que está conformado por la interrelación entre entidades animadas e inanimadas. Esto no quiere decir, como una lectura simplista podría hacer suponer, que la arena virtual se configure como preferencial; estas interrelaciones han de moverse de manera espuria y contaminada, localizándose en todos los espacios donde pueda ser útil/divertida. Así, hay que reconocer las prácticas de entretejimiento sobre las que se basan los networking sabiendo que cada networking es (re)creado a través de la interconexión de otras redes preexistentes.

Escuchar múltiples voces, así como las incoherencias entre sus discursos, en lugar de hablar para, como, a través de, con la pretensión de crear un discurso coherente. "Evitar una representación superficial de la 'diversidad' así como una 'unidad ontológica' (...). [E]n lugar de limitarse a construir sentidos, dejar que éstos puedan ser reescritos, (re)producidos y re/deconstruidos como actividad fundamentalmente relacional" (Clark, en Biglia et al. 2005, 17). Esto nos permite reconocer que la política es relación y que se puede trabajar conjuntamente, sin esperar encontrar acuerdos metodológicos o teóricos comunes, es decir, "no necesitamos apoyarnos en un único modelo de comunicación, un único modelo de razonamiento, una única noción del sujeto antes de ser capaces de actuar" (Butler 2001, 87).

De hecho, las subjetividades construidas como mujeres tienen mucha experiencia asociativa, sólo hay que dar un salto hacia la potenciación de las redes. Los encuentros realizados en la tesis me han demostrado cómo en situaciones más desfavorecidas las redes han sido fundamentales. Para usar una expresión muy querida por mi amigo Jordi Bonet i Martí, las mallas de seguridad sociales son constituidas y compuestas básicamente por mujeres; reconocer y ensalzar su valor político es, a mi entender, un reto para el feminismo. Podemos constituirnos como multitudes queer irrepresentables en su monstruosidad, reconociendo y trabajando desde la "transversalidad de las relaciones de poder una diversidad de las potencias de la vida" (Preciado 2003).

Esto así porque: "Si algo surge de la complejidad de los movimientos actuales, es el descubrimiento de que las culturas no se pueden formar o determinar con una única mano o factor. Incluso las concepciones de cambio han cambiado. La revolución ha sido revolucionada" (Plant 1998, 51). 


\section{Apuntes finales}

I think that through feminism, women come to know themselves and each other, with all our potential, our strengths, our weakness, and we discover a freedom that we

keep on developing. (Julieta, en PGA 2001, 45)

Uno de los aprendizajes más estremecedores del trabajo de investigación que he realizado ha sido descubrir la falacia de representar a las mujeres de contextos económico-culturales considerados pobres como más sumisas a las prácticas patriarcales. Lo que he podido tocar con las manos es que las expresiones del heteropatriarcado son muy diferentes y aunque en unos contextos son más aparentes que en otros, la visibilidad de la opresión no está proporcionalmente relacionada con su potencia ni con la incapacidad de reacción Por esto, un debate colectivo sobre las maneras en las que aspectos diferenciales del heteropatriarcado han sido subvertidos en diferentes lugares, puede ser un aprendizaje extremadamente útil. Respecto a la identificación como feminista, las narraciones presentadas muestran que la mayoría de las activistas entrevistadas en Italia hacían referencia de alguna manera $u$ otra al feminismo para describirse. No obstante, algunas consideraban necesario ampliar los sentidos del feminismo y otras eran extremamente críticas con la vertiente institucional elitista del movimiento. Esta última crítica era fuertemente repetida por las chilenas entrevistadas que, aun manteniendo una actitud extremamente autónoma y reivindicativa de los derechos de las mujeres, asociaban frecuentemente el feminismo a sus representaciones heteropatriarcales. Esta tendencia se percibía también, aunque en menor medida, en algunas activistas del Estado español que, sin embargo, se reivindicaban en su mayoría feministas. Con estos ejemplos espero haber mostrado cómo el auto identificarse como feminista no depende simplemente de la asunción de unas propuestas políticas, sino que está estrictamente relacionado con los imaginarios colectivos y las experiencias culturales e individuales al respecto. En el contexto en cuestión, por ejemplo, la intersección de tres características ha marcado de manera diferencial las vivencias generizadas y la conciencia de género: las características y temporalidad de los gobiernos totalitarios que han sometido a las tres naciones, las particularidades y la historia de los movimientos sociales mixtos $\mathrm{y}$, finalmente, la historia específica del movimiento feminista.

De manera parecida, si hoy en día el feminismo da mucho que hablar, no es baladí preguntarse si esto redunda en un contexto más o menos feminista que hace unos años y qué sentido tiene esto. Entre las expertas hay quienes sostienen que el feminismo es uno de los movimientos sociales más exitosos habiendo producido cambios sociales, políticos y culturales (Halberstam 2012). Sin embargo, otras nos recuerdan que, si bien el estatus de las mujeres ha mejorado muchísimo en los pasados 150 años, la revolución que llevaría a la igualdad aún no se ha producido (Lorber 2000). Siguiendo a bell hooks (1984/2000), los éxitos del movimiento han sido principalmente circunscritos a obtener reformas, en lugar de producir una real disminución del sexismo y la opresión "sexist values and assumption remain intact, not to mention racist values among some white feminists" (hooks 1984/2000, 159). Así, la institucionalización de una parte del feminismo y la transversalización de algunas de sus peticiones han influenciado las retoricas políticas (Walby 2002) con una mayor atención hacia el uso de lenguaje sexista o homofóbico (Mills 2008). Sin embargo, asistimos a la cooptación de muchas 
reivindicaciones feministas (Motta et al. 2011) vaciándolas de sus contenidos políticos. Es más, “[e]l eco mediático [que está teniendo el feminismo] puede crear el espejismo de igualdad (...) la sensación de que si se habla tanto, tal vez no haga falta seguir luchando" (Castejón 2018). Esto es particularmente peligroso teniendo en cuenta que los innegables avances producidos por los feminismos producen efectos muy diferenciales en los sujetos dependiendo de sus posicionamientos interseccionales (Walby 2011).

En este sentido es fundamental apostar por una política del networking que permita la colaboración feminista entre sujetos minorizados y vulnerabilizados independientemente de su adscripción de género o de sus autoidentificaciones como feministas. Siguiendo esta idea, y como conclusión de este texto, quiero compartir este patchwork (Biglia y Bonet-Martí 2009) realizado a partir de las propuestas de las activistas entrevistadas sobre como ponemos entender y corporeizar el feminismo para que se vaya ampliando desde otros posicionamientos situados.

Revindicar derechos de mujeres, siempre (Marta), no [es] la lucha (...) para hacerse iguales al hombre, sino para devenir alguna otra cosa (GrIt). [lo que más me gusta del feminismo] es el darte cuenta de ti misma, de tu cuerpo, de cómo hacer para transformar ciertas cosas (Roberta). [Por esto el feminismo para mí ha significado] haber empezado a pensar en mis contradicciones, en lo que tenía que soportar no obstante no me daba cuenta, en la manera como vivo en el mundo como mujer, así como en lo que yo como mujer tengo de bonito y de fuerte (Federica) [Así] me considero feminista porque me parece una lucha principal dentro de la amplia gama de luchas que puedes elegir (...), una lucha que hay que llevarla... (...) a todos los niveles, cada cual como puede (Verónica) [Dado que] la idea de ser feminista es también la idea de intentar pensar las cosas que haces traspasando los límites del pensamiento masculino (GrIt). [De hecho] desde el feminismo (...) hubo un movimiento social hecho de prácticas y de teoría (...) se pudo dar alternativas y propuestas interesantes que no son únicas pero que enriquecen y ayudan a trasformar (Micaela) [Es en este sentido que] me considero feminista porque no acepto el rol que me es impuesto socialmente como diferencia de género (Claudia) [O sea], yo creo que soy feminista porque primero soy mujer, me asumo como mujer. Segundo porque creo que lo que a nosotras nos falta es estimular el desarrollo de la organización de la mujer (...). Me declaro feminista porque (...) creo que nosotras tenemos que asumir el género (...). El feminismo se desarrollará a medida que nosotras ganemos nuestros espacios. (Gr2Ch)

\section{Referencias}

Alexander, M., y Mohanty, C., 2004. Genealogías, legados, movimientos. En: Traficantes de sueños, ed., Otras inapropiables: Feminismos desde las fronteras [en línea]. Madrid: Traficantes de sueños, 137-184. Disponible en: https://www.traficantes.net/sites/default/files/pdfs/Otras\%20inapropiablesTdS.pdf [Con acceso el 13 de enero de 2020].

Balasch, M., Callén, B., y Montenegro, M., 2005. Formes d'acció política no identitàries. En: Col-lectiu Investigacció, ed., Recerca activista i moviments socials. Barcelona: El viejo topo, $57-71$.

Balasch, M., y Montenegro, M., 2003. Una lectura articulatoria de los movimientos sociales: implicaciones para una política no confrontacional. Encuentros de Psicología Social, 1(3), 311-315. 
Bel Bravo, M.A., 2004. Siglo XXI: el reto de ser mujer. Hospitalidad ESDAI [en línea], 5, 7-20. Disponible en:

http://revistas.up.edu.mx/index.php/ESDAI/article/view/1328 [Con acceso el 13 de enero de 2020].

Biglia, B., 2005. Narrativas de mujeres sobre las relaciones de género en los movimientos sociales mixtos [en línea]. Tesis de Doctorado. Universidad de Barcelona. Colección Arxiu de Tesis de la Fundació Jaume Bofill. Disponible en:

https://www.fbofill.cat/publicacions/narrativas-de-mujeres-sobre-las-relacionesde-genero-en-los-movimientos-sociales?lg=es [Con acceso el 13 de enero de 2020].

Biglia, B., 2007. Teorías ¿sobre/para/desde/en/por? los MS. Àgora Revista de Ciencias Sociales, 17, 83-102.

Biglia, B., et al., 2005. Feminisms and Activisms: Reflections on the politics of writing and the editorial process. Annual Review of Critical Psychology, 4, 9-24.

Biglia, B., y Bonet-Martí, J., 2009. La construcción de narrativas como método de investigación psico-social. Prácticas de escritura compartida. Forum: Qualitative Social Research [en línea], 10(1). Disponible en: http://doi.org/10.17169/fqs$\underline{\text { 10.1.1225 }}$ [Con acceso el 13 de enero de 2020].

Biglia, B., y Bonet-Martí, J., 2017. DIY: Towards feminist methodological practices in social research. Annual Review of Critical Psychology [en línea], 13, 1-16. Disponible en: https://thediscourseunit.files.wordpress.com/2017/08/arcpbarbarab.pdf [Con acceso el 13 de enero de 2020].

Borio, G., Pozzi, F., y Roggero, G., 2004. La conivestigación como acción política. En: M. Malo, ed., Nociones comunes: Experiencias y ensayos entre investigación y militancia [en línea]. Madrid: Traficantes de sueños, 67-78. Disponible en:

https://www.traficantes.net/sites/default/files/pdfs/Nociones\%20comunesTdS.pdf [Con acceso el 13 de enero de 2020].

Bosch Fiol, E., et al., 2001. Feminismo social y feminismo académico. Mujeres en red [en línea]. Disponible en: http://www.nodo50.org/mujeresred/feminismo-ebvf.html [Con acceso el 13 de enero de 2020].

Brah, A., 2004. Diferencia, diversidad, diferenciación. En: Traficantes de sueños, ed., Otras inapropiables: Feminismos desde las fronteras [en línea]. Madrid: Traficantes de sueños, 107-136. Disponible en:

https://www.traficantes.net/sites/default/files/pdfs/Otras\%20inapropiablesTdS.pdf [Con acceso el 13 de enero de 2020].

Burman, E., 2003. Taking women's voices: The psychological politics of feminisation. Psychology of Women Section Review [en línea], 6(1), 3-21. Ponencia presentada en British Psychological Society Psychology of Women section Conference, University at Northampton. Disponible en:

https://ericaburmancom.files.wordpress.com/2013/01/2004-powsr-takingwomens-voices.doc [Con acceso el 13 de enero de 2020].

Butler, J., 1990. Gender Trouble: Feminism and Subversion of Identity. Nueva York: Routledge. 
Butler, J., 2001. Encuentros transformadores. En: E. Beck-Gernsheim, J. Butler y L. Puigvert, eds., Mujeres y transformaciones sociales. Barcelona: El Roure, 77-91.

Castejón, M., 2018. Feminismos y épica mediática: ¿2017, año de inflexión? Eldiario.es [en línea], 2 de enero. Disponible en https://www.eldiario.es/pikara/Feminismosepica-mediatica-ano-inflexion 6 723787633.html [Con acceso el 13 de enero de 2020].

Cirant, E., 2005. Io non sono femminista ma... Immagini di giovani donne nello specchio incrinato dell 'identità di genere'. En: E. Ruspini, ed., Donne e uomini che cambiano: Relazioni di genere, identità sessuali e mutamento sociale. Milán: Guerini, 91-120.

Cooper, B., 1995. The politics of diference and Women's Association in Niger: Of "prostitutes", the public and politics. Signs [en línea], 20(4), 851-882. https://doi.org/10.1086/495024 [Con acceso el 13 de enero de 2020].

Davies, M., ed., 1987. Third World, Second Sex. Londres: Zed books.

De Miguel, A., 1995. Los feminismos. En: C. Amorós, ed., Diez palabras clave sobre mujer. Pamplona: Verbo Divino, 247-293.

Escalera Karakola, 2004. Prólogo. En: Traficantes de sueños, ed., Otras inapropiables: Feminismos desde las fronteras [en línea]. Madrid, Traficantes de sueños. Disponible en: https://www.traficantes.net/sites/default/files/pdfs/Otras\%20inapropiablesTdS.pdf [Con acceso el 13 de enero de 2020].

Ferree, M., y Roth, S., 1998. Gender, Class, and the Interaction between Social Movements: A Strike of West Berlin Day Care Workers. Gender and Society [en línea], 12(6), 626-648. Disponible en: https://doi.org/10.1177/089124398012006003 [Con acceso el 13 de enero de 2020].

Fraire, M., y Frabotta, M., 2002. Introduzione. En: M. Fraire, ed., Lessico politico delle donne: teorie del Femminismo. Milán: Franco Angeli, 9-21.

Gamble, S., 2001. Postfeminism. En: S. Gamble, ed., The Routledge Companion to Feminism and Postfeminism. Nueva York/Londres: Routledge.

Ganser, D., 2005. NATO's Secret Armies: Operation Gladio and Terrorism in Western Europe [en línea]. Londres/Nueva York: Routledge. Disponible en: https://doi.org/10.4324/9780203017777 [Con acceso el 13 de enero de 2020].

García Dauder, S., y Romero Bachiller, C., 2002. Rompiendo viejos dualismos: (im)posibilidades de la articulación. Athenea [en línea], 2, 1-20. https://doi.org/10.5565/rev/athenea.51 [Con acceso el 13 de enero de 2020].

Gómez, L., 2004. Subjetivación y Feminismo: Analisis de un manifiesto politico. Athenea [en línea], 5, 1-27. Disponible en: https://doi.org/10.5565/rev/athenead/v1n5.119 [Con acceso el 13 de enero de 2020].

Halberstam, J., 2012. Whither Feminism? Gender and the New Normal. LA Review of Books [en línea], 7 de octubre. Disponible en:

https://areviewofbooks.org/article/whither-feminism-gender-and-the-newnormal/ [Con acceso el 22 de enero de 2013]. 
hooks, b., 2000. Feminist Theory: From Margin to Centre. $2^{\mathrm{a}}$ ed. Londres: Pluto Press. (Publicado originalmente en 1984).

Il colpo della Strega, ed., 1995. Dalle donne in politica alla politica delle donne. Appartenenza politica, appartenenza di genere dalla resistenza al neofemminismo [en línea]. Roma: DIY. Disponible en https://akarho.noblogs.org/files/2012/08/CdS.seminario.1995.pdf [Con acceso el 13 de enero de 2020].

Klandermans, B., y de Werd, M., 2000. Group identification and political protest. En: S. Stryker, T. Owens y R. White, eds., Self, Identity and Social Movements. Minneapolis: University of Minnesota, 68-90.

Las Clorindas, 2002. Feminismo Autónomo. I parte: Introducción. Gataclora [en línea], 1. Disponible en: http://www.mujeresenred.net/spip.php?article303 [Con acceso el 13 de enero de 2020].

Lorber, J., 2000. Using Gender to Undo Gender: A Feminist Degendering Movement. Feminist Theory [en línea], 1(1), 79-95. https://doi.org/10.1177/14647000022229074 [Con acceso el 13 de enero de 2020].

Magaraggia, S., Martucci, C., y Pozzi, F., 2005. The great fresco painting of the Italian feminist movements. Annual Review of Critical Psychology, 4, 26-38.

Meyers, D., ed., 1997. Feminist Social Thought: A Reader. Nueva York/Londres: Routledge.

Mills, S., 2008. Language and Sexism. Cambridge University Press.

Motta, S., et al., 2011. Feminism, Women's Movements and Women in Movement. Interface, 3(2), 1-32.

Neuhouser, K. ,1995. “Worse Than Men”: Gendered Mobilization in an Urban Brazilian Squatter Settlement, 1971-91. Gender and Society [en línea], 9(1), 38-59. Disponible en: https://doi.org/10.1177/089124395009001003 [Con acceso el 13 de enero de 2020].

Nicholson, L., ed., 1997. The Second Wave: A Reader in Feminist Theory. Nueva York/Londres: Routledge.

Noonan, R., 1995. Women against the state: Political opportunities and collective action frames in Chile's transition to democracy. Sociological Forum [en línea], 10(1), 81111. Disponible en: https://doi.org/10.1007/BF02098565 [Con acceso el 13 de enero de 2020].

Peoples' Global Action, 2001. Desire for Change: Women on the Frontline of Global Resistance [en línea]. Londres: PGA. Disponible en:

https://www.nadir.org/nadir/initiativ/agp/gender/desire/desireforchange.pdf [Con acceso el 13 de enero de 2020].

Piedra Guillén, N., 2003 Feminismo y postmodernidad: entre el ser para sí o el ser para los otros. Revista de Ciencias Sociales [en línea], 4(102), 43-55. Disponible en: https://revistacienciassociales.ucr.ac.cr/images/revistas/RCS101_102/03PIEDRA . pdf [Con acceso el 13 de enero de 2020]. 
Plant, S., 1998. Ceros + Unos. Barcelona: Destino.

Preciado, B., 2003. Multitudes queer. Multitudes [en línea], 12, 17-25. Disponible en: https://doi.org/10.3917/mult.012.0017 [Con acceso el 13 de enero de 2020].

Reicher, S., 2004. The context of social identity: Domination, resistance and change. Political Psychology [en línea], 25(6), 921-945. Disponible en: https://doi.org/10.1111/j.1467-9221.2004.00403.x [Con acceso el 13 de enero de 2020].

Roseneil, S., 2000. Common Women, Uncommon Practices: The Queer Feminism of Greenham. Londres/Nueva York: Cassell.

Serrano, M., y López, S., 2003. Positions, Situations, Short-Circuits: La Eskalera Karakola, A Deliberate Space [en línea]. Ponencia presentado en Gender and Power in the New Europe, the $5^{\text {th }}$ European Feminist Research Conference. Universidad de Lund, 20-24 agosto. Disponible en:

https://cdn.atria.nl/epublications/2003/Gender and power/5thfeminist/paper 305 303.pdf [Con acceso el 13 de enero de 2020].

Sum, N.L., 2000. From politics of identity to politics of complexity: a possible research agenda for feminist politics/movements across time and space. En: S. Ahmed et al., eds., Transformations: Thinking through Feminism. Nueva York/Londres: Routledge, 131-144.

Taylor, V., 2000. Utopian vision: Engaged sociologies for the 21st century. Contemporary Sociology [en línea], 29(1), 219-230. Disponible en: https://doi.org/10.2307/2654946 [Con acceso el 13 de enero de 2020].

Walby, S., 2002. Feminism in a global era. Economy and Society [en línea], 31(4), 533-557.

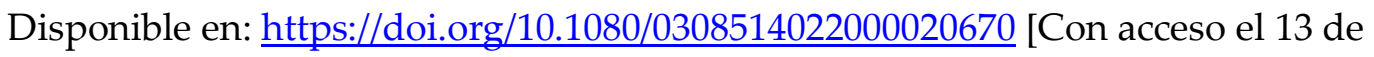
enero de 2020].

Walby, S., 2011. The Future of Feminism. Cambridge: Polity Press.

Waylen, G., 1994. Women and Democratization: Conceptualising gender relation in transition politics. World Politics [en línea], 46(3), 327-354. Disponible en: https://doi.org/10.2307/2950685 [Con acceso el 13 de enero de 2020].

Young, I.M., 1990. The ideal of community and the politics of difference. En: L.J. Nicholson, ed., Feminism/Postmodernism. Nueva York/Londres: Routledge.

Yuval-Davis, N., 1993. Beyond difference: Women and Coalition Politics. En: M. Kennedy, C. Lubelska y V. Walsh, eds., Making Connections: Women's Studies, Women's Movements, Women's Life. Londres: Taylor and Francis, 3-10. 